\title{
Role of Ehrlichiosis in Canine Pup Mortality
}

\author{
K. Arulanandam, R. Sridhar, T. Mohanapriya* and C. Balachandran \\ Department of Veterinary Pathology, Madras Veterinary College, Chennai - 600007, India \\ *Corresponding author
}

\section{A B S T R A C T}

Pup mortality during parturition and also in the neonatal period or even later is a significant problem that is poorly documented in the veterinary

\section{Keywords}

E.canis, Pup mortality, Morulae, Splenic plasmacytosis

\section{Article Info}

Accepted:

12 May 2020

Available Online:

10 June 2020 literature. Out of 77 pups died during the one year period, four (5.19 percent) ehrlichiosis belonged to 27 to 90 days age. Anorexia, dullness, vomiting, diarrhoea, icterus, and petechiae on the ventral abdomen were observed. Morulae were observed in monocytes/macrophages in the blood and splenic impression smears. Leucopaenia, monocytosis, azotaemia, hypoproteinemia and hypoalbuminaemia were observed. Haemoperitoneum, haemothorax, hepatomegaly, splenomegaly, thickening of gall bladder, pulmonary edema and haemorrhage, renal cortical discoloration and prominent cortico-medullary junction were observed. Gastric oedema, subacute enteritis, centrilobular necrosis, degeneration of hepatocytes, biliary hyperplasia, myocarditis, interstitial nephritis, lymph node and splenic plasmacytosis were also observed.

\section{Introduction}

Among the organisms known to cause illness in dogs, E. canis was considered to be the most important agent worldwide, due to the potential severity of the illness and the difficulty in curing the patients (CFSPH, 2013). Price et al. (1987) reported that canine ehrlichiosis was observed between the age groups of 3 months to 13 years. Chandrasekar et al. (2002) reported lymphadenopathy, vomiting, pyrexia, anorexia, congested mucous membrane, epistaxis, selective appetite, diarrhoea, petechiae and ecchymosis as the most common clinical signs in canine ehrlichiosis. In a study of 10 anaemic dogs with ehrlichiosis, haematology revealed moderate to non-regenerative microcytic normochromic anaemia and severe thrombocytopaenia. Eighty percent of the cases had WBC count lower than $6.0 \times 10^{3} \mu \mathrm{L}$. E. canis infected dogs showed petechial and 
ecchymotic haemorrhages on the serosal and mucosal surface of most organs including the nasal cavity, lung, kidney, urinary bladder, gastrointestinal tract and subcutaneous tissue. Generalized lymphadenomegaly, splenomegaly and hepatomegaly were mostly seen during the acute phase (Chandrasekar et al., 2002).

Perivascular plasma cell infiltration was observed in numerous organs like the lungs, brain, meninges, kidneys, lymph node, bone marrow, spleen and sometimes skin or mucosa. The degree of plasma cell infiltrate appeared to increase in chronically infected dogs. In the central nervous system, there was a multifocal, non-suppurative meningoencephalitis which involved the brainstem, mid brain and cerebral cortex (Neer, 1996). Moderate portal fibrosis with bile duct proliferation, dilatation of thin-walled vessels and subtle portal to portal bridging fibrosis (Mylonakis et al., 2010). Along with morulae, thrombocytopaenia, microcytic normochromic anaemia and mild leucopaenia were the most common haematological abnormalities in ehrlichiosis affected dogs (Shah et al., 2010, CFSPH, 2013).

\section{Materials and Methods}

Puppies less than 6 months old, admitted to the Small Animal Clinics were subjected to a thorough clinical examination with tentative diagnosis of Ehrlichiosis. The pups which succumbed to death inspite of treatment were brought to Department of Veterinary Pathology, MVC, Chennai. Detailed postmortem examinations of the dead puppies were conducted. Peripheral blood smear and spleen impression smears were collected to check for the presence of morulae in monocytes/macrophages. Tissue samples from various organs were collected in 10 percent formalin for histopathology.

\section{Results and Discussion}

Out of 77 pups, four (5.19 percent) were positive for ehrlichiosis. These were a 48 days old male Saint Bernard, a 90 days old female Golden retriever and two of female Labrador retriever amongst which one was 60 days and another was 27 days old. Respiratory distress and hemoptysis were observed in one pup. Anorexia, dullness and petechiae on the ventral side of the abdomen and icterus in two pups, oedema of the hind limbs and ascites were observed in one and vomiting and diarrhoea were noticed in another one. Peripheral blood smears revealed morulae in two pups whereas splenic impression also revealed morulae. Visible mucous membranes were icteric in two, blanched in one and pale pink in one pup. Oedema of hind limbs was observed in one pup. Petechiae and ecchymosis of the ventral abdomen were observed in two pups. There was no evidence of ticks in all the four. Subcutaneous tissue was icteric in two pups. Peripheral lymph nodes were enlarged and oedematous. Stomach revealed paintbrush haemorrhages on the serosa and severe oedematous mucosa in one case. Intestine showed segments of $D$. caninum mixed with dirty yellow feed contents in one case. Liver showed accentuated lobular pattern with mottling in two pups, pale yellow and mottled in one and pale in another one. Trachea contained frothy fluid. Lungs showed oedema, multifocal petechiae and ecchymosis in two pups and small pieces of lung sank in water. Brown interspersed with pink areas in one and numerous patchy grey white areas interspersed with red brown areas in another were observed in lungs. Spleen was enlarged, dark purple with round borders in one, sharp borders with yellow encrustations Kidneys showed grey brown discoloration of the cortical surface. Meningeal vessels of brain were congested in all the cases. Stomach revealed submucosal congestion and mild 
desquamation of the mucosal epithelium. Severe submucosal oedema was observed in one pup. Intestine showed blunting of villi, desquamation and dilated crypts with hyperplasia in three pups and severe lymphoplasmacytic infiltration of the mucosa in one pup.

Liver showed centrilobular necrosis, fatty degeneration of hepatocytes which appeared to form fatty cysts and compressing central veins dilatation of sinusoids and massive infiltration of lymphoplasmacytic cells around the central vein and portal areas in all the four cases. Macrophages containing pigments in two cases and bile stasis with mild bile duct hyperplasia were recorded in two cases. Heart showed diffuse vacuolar degeneration and waviness of the myocardial fibres in two cases and focal mononuclear cell infiltration in another case. Lungs revealed multifocal areas of hyperaemia, oedema, emphysema, haemorrhage and perivascular and mild peribronchial infiltration with mononuclear cells in all the four cases. Blood vessels were occluded with erythrocytes and thrombus in

\section{Lymph node - Plasmacytosis}

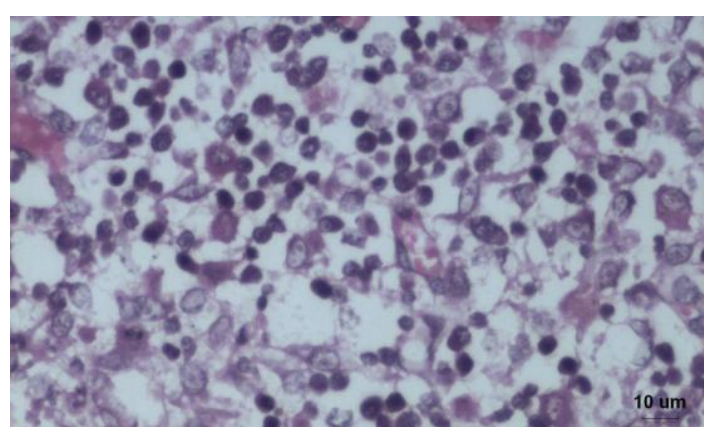

Out of 77 cases, four $(5.19$ percent $)$ ehrlichiosis belonged to 27 to 90 days age. However, Price et al. (1987) observed that the disease occured between 3 months to 9 years of age. Anorexia, dullness, vomiting, diarrhoea, icterus, and petechiae on the ventral abdomen were also observed by one case. Scattered hemosiderin pigments were observed in one case. Severe neutrophilic infiltration in the alveoli was observed in one case. Lymph node showed altered architecture, lymphoid depletion, atrophy of paracortical areas, reticular hyperplasia and plasmacytosis in all the four cases. Focal interstitial oedema and severe congestion in one case and focal interstitial proliferation in another case were observed. Kidney showed multifocal, expanding the interstitium at the corticomedllary junction and radiating into the medulla and cortex with admixture of plasma cells with a fewer lymphocytes in three cases. Multifocal congestion with haemorrhage was seen in one case. Degeneration and necrosis of the proximal tubules were observed in all the four cases. In two cases, a few glomerular atrophy and cystic dilatation were noticed. Bladder showed mild congestion in two cases. Spleen revealed mild to moderate accumulation of perivascular plasma cells, lymphoid cells depletion, reticulum hyperplasia and congestion in all the four cases.

\section{Spleen -E.canis - Morula - Monocyte}

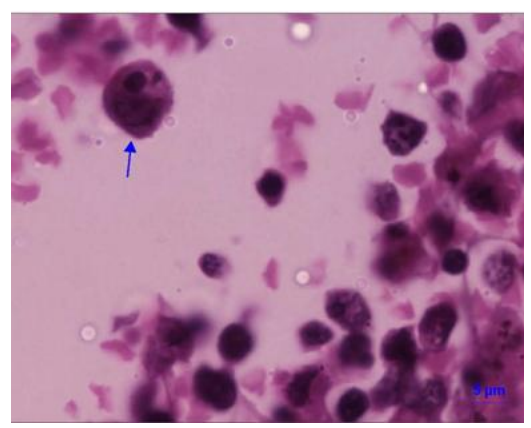

Chandrasekar et al. (2002). Peripheral blood smear showed morulae within monocytes in two cases and impressions from spleen in all the four pups. Similar observations were also made by Mohanapriya (2012). Leucopaenia with monocytosis in one case and azotaemia, hypoproteinemia and hypoalbuminaemia in 
two cases were observed (Shah et al., 2010). Icteric visible mucous membranes in two and blanched in one case, hind limb oedema in one, and petechiae and ecchymosis of the ventral abdomen in two cases were observed. However, there was no evidence of ticks in all the four cases. Accumulation of serosanguineous fluid in the abdominal and thoracic cavities, hepatomegaly, splenomegaly, thickening of gall bladder, pulmonary oedema and haemorrhage, renal cortical discoloration in two cases and prominent cortico medullary junction in one case observed were in concurrence with Chandrasekar et al. (2002). Gastric oedema and subacute enteritis, centrilobular necrosis and fatty degeneration of hepatocytes and biliary stasis and hyperplasia observed were in concurrence with Mylonakis et al. (2010). Myocardial degeneration, myocarditis and interstitial nephritis, lymph node and splenic plasmocytosis observed were similar to those of Neer (1996) who reported that perivascular plasma cell infiltration was the predominant change observed in various organs in E. canis infected dogs and the degree of plasma cell infiltrate appeared to increase in clinically infected dogs.

In conclusion, the presence of morulae in the monocytes/macrophages is the important pathognomonic changes diagnosed in the ehrlichiosis of these pups. This paper suggested various clinical signs, clinicpathological and histopathological in different organs caused by Ehrlichia canis, which leads to mortality in canine pups. This study gave a clear evidence of inevitable role of ehrlichiosis in canine pup mortality.

\section{References}

Chandrasekar M, Nambi AP, Ramprabhu R, Dhanapalan P. 2002. Epizootiological studies on canine ehrlichiosis. Indian Veterinary Journal, 79: 1311 -1312.

Meneses A. 1995. First report of canine ehrlichiosis in Costa Rica. Veterinary Record, 137: 46-47.

Mohanapriya T. 2012. Pathology of canine splenic disorders. 2012 M.V.Sc thesis approved by Tamil Nadu Veterinary and Animal Sciences University, Chennai, India.

Mylonakis ME, Kritsepi-Konstantinou M, Dumler JS, Diniz PPVP, Day MJ, Siarkou VI, et al. 2010. Severe hepatitis associated with acute Ehrlichia canis infection in a dog. Journal of Veterinary Internal Medicine, 24: 633-638.

Neer TM. 1996. Clinical approach to splenomegaly in dogs and cats. Compendium on Continuing Education for the Practising Veterinarian. 18:35-48

Price JE, Sayer PD and Dolan TT. 1987. Improved clinical approach to the diagnosis of canine ehrlichiosis. Tropical Animal Health Production, 19: 1-8.

Shah SA, Sood NK, Uppal SK and Gupta K. 2010. Ehrlichiosis in anemic and thrombocytopenic dog in Punjab, India. Indian Journal of Veterinary Pathology, 34: 36-37.

www.cfsph.iastate.edu/Factsheets/pdfs/hook worms.pdf.ehrlichiosis.pdf. 2013 1-14. (Accessed on 15.07.2013).

\section{How to cite this article:}

Arulanandam, K., R. Sridhar, T. Mohanapriya and Balachandran, C. 2020. Role of Ehrlichiosis in Canine Pup Mortality. Int.J.Curr.Microbiol.App.Sci. 9(06): 4232-4235. doi: https://doi.org/10.20546/ijcmas.2020.906.496 